\title{
The Health and Retirement Study Harmonized Cognitive Assessment Protocol Project: Study Design and Methods
}

\author{
Kenneth M. Langa ${ }^{\text {a-d }}$ Lindsay H. Ryan ${ }^{c}$ Ryan J. McCammon ${ }^{c}$ \\ Richard N. Jones ${ }^{e}$ Jennifer J. Manly ${ }^{f}$ Deborah A. Levine ${ }^{a, d, g}$ \\ Amanda Sonnega $^{c}$ Madeline Farron ${ }^{a}$ David R. Weir ${ }^{c}$ \\ aDivision of General Medicine, Department of Internal Medicine, University of Michigan Medical School, Ann \\ Arbor, MI, USA; ${ }^{b}$ Veterans Affairs Center for Clinical Management Research, Ann Arbor, MI, USA; ${ }^{C}$ Institute for Social

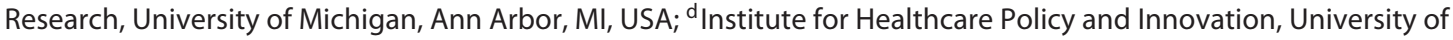 \\ Michigan, Ann Arbor, MI, USA; ${ }^{e}$ Department of Psychiatry and Human Behavior and Neurology, Brown University

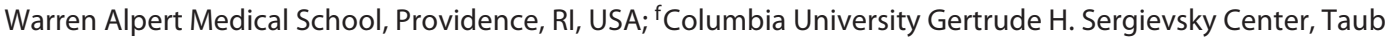 \\ Institute for Research in Aging and Alzheimer's disease, New York, NY, USA; 9 Department of Neurology and Stroke \\ Program, University of Michigan, Ann Arbor, MI, USA
}

\author{
Keywords \\ Population-based research · Dementia risk factors · \\ Dementia costs · International comparisons · \\ Cohort studies
}

\begin{abstract}
Introduction: The Harmonized Cognitive Assessment Protocol (HCAP) Project is a substudy within the Health and Retirement Study (HRS), an ongoing nationally representative panel study of about 20,000 adults aged 51 or older in the United States. The HCAP is part of an international research collaboration funded by the National Institute on Aging to better measure and identify cognitive impairment and dementia in representative population-based samples of older adults, in the context of ongoing longitudinal studies of aging in high-, middle-, and low-income countries around the world. Methods: The HCAP cognitive test battery was designed to measure a range of key cognitive domains affected by cognitive aging (including attention, memory, executive function, language, and visuospatial function) and to
\end{abstract}

allow harmonization and comparisons to other studies in the United States and around the world. The HCAP included a pair of in-person interviews, one with the target HRS respondent (a randomly selected HRS sample member, aged $65+$ ) that lasted approximately $1 \mathrm{~h}$ and one with an informant nominated by the respondent that lasted approximately 20 min. The final HRS HCAP sample included 3,496 study subjects, representing a $79 \%$ response rate among those invited to participate. Conclusion: Linking detailed HCAP cognitive assessments to the wealth of available longitudinal HRS data on cognition, health, biomarkers, genetics, health care utilization, informal care, and economic resources and behavior will provide unique and expanded opportunities to study cognitive impairment and dementia in a nationally representative US population-based sample. The fielding of similar HCAP projects in multiple countries around the world will provide additional opportunities to study international differences in the prevalence, incidence, and outcomes of dementia globally with comparable data. Like all HRS data, HCAP data are publicly available at no cost to researchers.

\section{KARGER}

(c) 2019 S. Karger AG, Basel

E-Mail karger@karger.com

www.karger.com/ned
Kenneth M. Langa, MD, $\mathrm{PhD}$

Division of General Medicine, Department of Internal Medicine

University of Michigan Medical School, 2800 Plymouth Road

Building 16, Room 444E, Ann Arbor, MI 48109-2800 (USA)

E-Mail klanga@umich.edu 


\section{Introduction}

Dementia, a decline in memory and other cognitive functions severe enough to cause disability in daily activities, has a large and growing impact on older adults, their families, and on government programs in the United States and around the world. In 2015, about 5 million adults in the United States and $>46$ million around the world had dementia due to Alzheimer's disease (AD) and other AD-related dementias [1]. The economic impact of dementia, including a large burden of unpaid family caregiving, has been estimated at USD 200 billion per year in the United States [2] and USD 800 billion worldwide [1], which is greater than the economic impact of other common chronic diseases, such as heart disease and cancer. The incidence of dementia rises sharply at older ages, so the growth in the worldwide elderly population in the decades ahead is expected to lead to a near tripling of dementia cases by 2050 absent new interventions to prevent or delay dementia onset [1].

Because of this large and growing impact of dementia, governments, policy-makers, and researchers around the world are prioritizing data collection on individuals and populations to better understand, address, and track the current and future impact of the dementia epidemic. In the United States, the Health and Retirement Study (HRS) has been a leading data resource for this purpose [3]. The HRS began collecting data on a national sample of U.S. adults in 1992 and has now collected up to 25 years of biennial follow-up data on $>43,000$ individuals aged 51 or older. The HRS has also become the model for a large network of harmonized longitudinal studies of aging with similar study designs and content in $>40$ countries around the world [4].

In 2002-2003, the first HRS substudy focused on cognitive impairment and dementia-the Aging, Demographics, and Memory Study (ADAMS)-provided the first estimates of dementia incidence and prevalence in a nationally representative sample in the United States [5]. With linkage to the wide range of longitudinal data on health, wealth, and family in the core HRS, research using these data has provided important insights on the risk factors and outcomes of dementia in the United States. The new Harmonized Cognitive Assessment Protocol (HCAP) project builds on the experience and methods of the HRS and ADAMS for studying cognition, cognitive decline, and dementia in a representative population-based sample. By collaborating and harmonizing with multiple international partners of the HRS, the HCAP will establish an international data resource to better understand and compare the causes, consequences, and trends in dementia in the United States and around the world, as key factors such as levels of educational attainment, the prevalence of cardiovascular risk factors, and life expectancy vary across countries, and over time. In this article, we describe the design and methods of the HCAP, report basic HRS-HCAP sample characteristics, illustrate potential uses of the data, and provide information on obtaining the HCAP data.

\section{The HCAP}

\section{Main Goals of the HCAP Project}

The main goals of the HCAP project are to (1) create a new HRS data resource to better assess the current and future trends in the determinants, prevalence, and costs and consequences of mild cognitive impairment (MCI) and dementia in the United States and (2) facilitate international harmonization of cognitive measurement and cross-national multidisciplinary studies of the determinants, prevalence, and impact of MCI and dementia, now and in the future. Our strategies to achieve these goals are to (1) administer an expanded battery of cognitive tests and informant interviews to a random subsample of HRS respondents aged 65 and older and (2) collaborate with research teams from the HRS international family of studies to develop and implement similar cognitive testing protocols across studies.

\section{Lessons from ADAMS}

The ADAMS embedded a detailed collection of cognitive data and a clinical interview for the diagnostic classification of cognitive impairment or dementia in a subsample of HRS respondents and provided unique opportunities for researchers to study the risk factors and outcomes of dementia in the growing population of older adults. However, a key limitation of the ADAMS was its high cost resulting from a 3- to 4-h in-home assessment administered by specialized personnel (a nurse and neuropsychology technician). Based on HRS experience administering cognitive tests as part of the CogUSA project [6], the HCAP implemented a more limited 1-h cognitive assessment using a computer-assisted personal interview by a trained survey interviewer, significantly reducing the cost per assessment and allowing for a sample that is 4 times larger than ADAMS. The much larger sample size will significantly increase the statistical power for analyses using these new data compared to the ADAMS sample, opening up greater opportunities 
for subanalyses, such as the relationship of race/ethnicity, education, comorbidities, and geographic location to dementia risk.

\section{International Collaboration}

The HCAP was specifically designed to facilitate crossnational comparisons of the prevalence and trends of dementia in aging populations around the world. The HCAP resulted from ongoing collaborations and consultations with investigators from many of the HRS international family of studies, as well as leading experts in dementia epidemiology, including Martin Prince, principal investigator of the 10/66 Dementia Research Group [7], Hugh Hendrie, PI of the Indianapolis-Ibadan Dementia Project [8], and Carol Brayne, PI of the UK Cognitive Function and Ageing Study [9]. The HRS and National Institute on Aging (NIA) organized a number of meetings among this multidisciplinary group of collaborators in the years leading up to the first HCAP field period, resulting in close collaboration in developing, pilot testing, and fielding the HCAP. The HCAP has been implemented in the following HRS international sister studies: Mexican Health and Aging Study [10], Longitudinal Aging Study in India [11], English Longitudinal Study of Ageing [12], Chinese Health and Retirement Longitudinal Study [13], and the Health and Aging in Africa: A Longitudinal Study of an INDEPTH Community in South Africa [14]. Two additional studies, the Survey of Health and Retirement in Europe [15] and the Korean Longitudinal Study of Aging $[16,17]$ will be fielding HCAP studies in the next 2 years.

Using the HRS and ADAMS as the Foundation for the HCAP Project

The HRS is a nationally representative, prospective cohort study of U.S. adults that began collecting data in 1992 [3]. The HRS core survey is a biennial interview designed to investigate the health, social, and economic implications of aging in America. The initial 1992 cohort was born 1931-1941; a second cohort was added in 1993, capturing those born before 1924. In 1998, 2 new cohorts were enrolled to bridge the study age gaps for U.S. adults age 51 and older. The HRS adds to the sample new birth cohorts every 6 years: the Early Baby Boomers (born 1948-1953) in 2004, the Mid Baby Boomers (born 19541959 ) in 2010, and the Late Baby Boomers (born 19601965 ) in 2016. Both members of a couple, regardless of the partner's age, are included in the sample. The HRS oversamples African-American and Hispanic households, and in 2010 the minority sample was further increased from a supplemental screening of the Early- and
Mid-Baby Boomer cohorts. The study administers all interviews and mail surveys in English and Spanish.

Since 2006, the HRS has used a mixed-mode design in which half of the core sample is randomly assigned to a face-to-face core interview enhanced with physical and biological measures and a psychosocial questionnaire, and the other half is assigned to a telephone core-only interview. Other key design features include use of proxy respondents for those who are unwilling or unable to complete the interview independently; continued follow-up for respondents who move in to nursing homes; and postmortem interviews with an informant after a respondent's death. In addition to the core biennial survey, HRS conducts a wide range of supplemental studies usually in the off years from the core. Finally, HRS data are linked at the individual level to several sources of administrative information including the Social Security Administration for information on earnings and benefits, the Centers for Medicare and Medicaid Services for health care claims information, and to the Veterans Administration for utilization of Veterans Administration health care services. At the employer level, information on employer-provided pension plans is obtained for businesses where respondents are or have been employed. Further details have been published previously [3] and are also available at the HRS website (http://hrs.isr.umich.edu). The HRS is funded by the NIA (U01 AG009740), with supplemental funding from the Social Security Administration, and conducted by the Institute for Social Research at the University of Michigan.

From its inception, the HRS has made cognition a focus of its measurement, including repeated administration of measures of episodic memory, attention, and orientation, as well as structured informant reports in proxy interviews. The ADAMS project conducted in-depth assessments for the diagnosis of cognitive impairment and dementia in a stratified subsample drawn from the HRS. The ADAMS was designed to produce a diagnostic determination consistent with that from a high-quality clinical research study; it had multiple diagnostic review steps, concluding with a consensus conference of neuropsychologists, neurologists, and other experts to adjudicate cases. When available, the study reviewed medical records, including imaging results.

As noted previously, the ADAMS provided the first national estimates of the prevalence and incidence of dementia and cognitive impairment from a U.S. population-representative sample [18-20]. The study also provided a valuable validation of the cognitive measures administered in the core HRS interview. Based on various statistical approaches to modeling the ADAMS diagnoses
Langa/Ryan/McCammon/Jones/Manly/ Levine/Sonnega/Farron/Weir 
as functions of HRS core measures, researchers have since been able to use the entire HRS sample to address important questions about the epidemiology and population impact of dementia. For example, the method of "equipercentile equating" based on the ADAMS MCI and dementia prevalence estimates was used to identify cutpoints on the HRS self- and proxy-cognition scales to create normal, $\mathrm{MCI}$, and dementia diagnostic groups for the full HRS sample [21]. Hurd et al. [2] used HRS cognition and functional limitation data to develop a 3-category ordered probit regression model to estimate the probability of normal, MCI, or dementia in ADAMS participants, separately for self- and proxy-respondents. Finally, Wu et al. [22] combined HRS data for both self- and proxy-respondents to develop a logistic regression model to predict the odds of dementia for ADAMS participants. A recent useful paper compared the sensitivity, specificity, and accuracy of these diagnostic algorithms using the ADAMS diagnosis as the reference standard and found that accuracy exceeded $90 \%$ for all 3 algorithms, when using sampling weights in the analyses [23].

Use of HRS measures that have been calibrated to the ADAMS diagnoses using the statistical methods described above has allowed a range of studies on key issues such as the monetary costs of dementia, including the large amount of unpaid care provided by family members [2], the impact of acute severe illness on cognitive decline [24], the impact of cardiovascular risk factors on dementia risk [25], and the relationship of dementia to the aggressiveness of care at the end of life and in the presence of advance directives [26, 27].

Data from the HRS and ADAMS are widely used and published. As of April 2019, the HRS bibliography (https:// hrs.isr.umich.edu/publications/biblio/) included 2,853 journal articles, 837 reports, 199 books or book chapters, and 502 master's theses or doctoral dissertations.

\section{HCAP Sample Selection and Response Rates}

As in the ADAMS, the HRS sample provided the sample frame for the HCAP. The HCAP sample was randomly selected from HRS panel respondents age 65 or older (birth year 1952 or earlier) who completed their 2016 core interview prior to eligibility for the HCAP. Study entry at age 65 was chosen (as compared to age 70 in ADAMS) to increase the likelihood of detecting early stages of cognitive decline. Age 65 also marks the age of Medicare eligibility, allowing access to medical records for those who consent to linkage, which includes about $90 \%$ of the HCAP sample. To ensure representation of both single and coupled households, sampling included random selection of
Table 1. HRS HCAP project response rates

\begin{tabular}{llll}
\hline $\begin{array}{l}\text { Age at 2016 } \\
\text { HRS interview }\end{array}$ & $\begin{array}{l}\text { Response } \\
\text { rate, \% }\end{array}$ & Race/ethnicity & $\begin{array}{l}\text { Response } \\
\text { rate, \% }\end{array}$ \\
\hline $65-74$ & 79 & White & 78 \\
$75-84$ & 80 & Black & 81 \\
$85+$ & 77 & $\begin{array}{l}\text { Hispanic } \\
\text { All others }\end{array}$ & 80 \\
& & 2016 HRS & Response \\
\hline Education, & Response & interview type & rate, \\
years & rate, \% & Self & 80 \\
\hline $0-11$ & 77 & Proxy & 62 \\
12 & 79 & & \\
$13-15$ & 79 & & \\
$16+$ & 81 & &
\end{tabular}

HRS, Health and Retirement Study; HCAP, Harmonized Cognitive Assessment Protocol.

one-half of all eligible single respondent households and random selection of one respondent from each eligible coupled household. The HCAP included an interview with an informant who was one of up to 3 individuals nominated by the respondent. Informant relationships with the respondent included spouse/partner (45\%), child (27\%), friend (12\%), sibling (4\%), grandchild (2\%), parent (1\%), neighbor $(0.9 \%)$, guardian $(0.2 \%)$, or other relationship (7\%). The mean number of years an informant reported knowing the respondent was 30 (SD 21; range 1-87). The HCAP was administered either in English (95\%), in Spanish $(5 \%)$, or both $(0.1 \%)$. The respondent interview took approximately $1 \mathrm{~h}$ and the informant interview about 20 min to complete. Like the HRS face-to-face interviews, the HCAP respondent and informant interviews took place in the respondent's home or residence and were administered by specially trained HRS interviewers.

Of 4,425 eligible cases, 3,496 completed the HCAP interview between June 2016 and October 2017 for a final response rate of $79 \%$. Table 1 shows that HCAP response rates were generally similar across age, race/ethnicity, sex, and education level but were somewhat lower for individuals who were represented by a proxy at the prior HRS interview. There were 149 cases where only an informant interview was collected when the HCAP respondent was not able to conduct an interview, but an informant was nominated and enrolled. A total of 313 cases were respondent only cases where an informant was not nominated by the respondent or where a nominated informant was not enrolled. Overall, $87 \%$ of HCAP cases included both a respondent and informant interview. 
Study subjects and their informants provided written informed consent to participate. The HRS and HCAP study protocols were approved by the University of Michigan Institutional Review Board.

\section{Selection of Tests for the HCAP Instrument}

To meet the multiple aims of the HCAP project, the key criteria for selection of the component cognitive tests were that they: (1) covered a range of cognitive domains in order to provide valid research diagnoses of MCI and dementia; (2) had substantial overlap with the initial ADAMS assessment (2002-2003) to facilitate comparisons to the earlier study; (3) could be administered by trained survey interviewers in the home in about $1 \mathrm{~h}$; (4) could be administered in comparable format by survey interviewers in other countries, including low-income countries, to promote harmonization across the HRS international family of surveys; (5) had broad credibility and acceptance within the scientific community; and (6) could be readministered longitudinally to identify incident cognitive impairment and dementia.

To identify the component tests that best fit these criteria, we reviewed the content of ADAMS, the Rush Religious Orders Study (ROS), and Memory and Aging Project (MAP) [28], as well as several other community-based studies of cognitive impairment and dementia, including the 10/66 dementia studies [7], the Indianapolis-Ibadan Dementia Project [8], and the UK Medical Research Council Cognitive Function and Ageing Study [9]. The core elements common to nearly all these prior studies were (a) Mini-Mental State Examination, (b) CERAD word list immediate and delayed recall, and (c) Semantic Fluency (Animal Naming Test). To evaluate the contribution of these and other tests to diagnosis, we conducted statistical analyses of data from ADAMS and the ROS/ MAP studies, with the assistance of David Bennett, the Principal Investigator of ROS/MAP. The ROS/MAP longitudinal studies include over 20,000 cognitive assessments using many of the same tests as ADAMS, with some significant additions.

After identifying common tests that covered a range of cognitive domains across ADAMS, ROS-MAP, and the other community-based studies noted above, we assessed the rates of missing data for these tests. Individuals with dementia are typically unable to complete some tests; they may express this directly, or the test administrator may exercise their own judgment and skip a test, or end the testing altogether before all tests are complete. For HCAP, our goal was to minimize the role of interviewer judgment to maximize consistency of administration across interviewers, so we prioritized tests with low rates of missing data.

We then assessed the predictive power of the individual tests for identifying those diagnosed with dementia in the ADAMS and ROS-MAP studies using a multivariate ordered probit model. Tests with the greatest predictive power were selected and included in the final HCAP protocol. More details regarding the HCAP test selection process are available at the HRS website [29].

\section{HCAP Respondent and Informant Measures}

Table 2 shows the full HCAP neuropsychological test battery administered to the respondent and the informant, listed in the order of administration. More detail on the specific tests and study protocol is available at the HRS website [30]. Both respondent and informant interviews were conducted by trained survey interviewers using computer-assisted personal interview technology.

Since data from the HCAP can be linked to the respondent's HRS data, the HCAP protocol excluded the measurement of some factors relevant to performance on cognitive tests because that information was already captured in the HRS core interview (e.g., a history of stroke). Although an abbreviated form of the CES-D depression assessment is administered in the HRS core interview, it was also administered at the end of the HCAP interview due to the potential impact of depressive symptoms on cognitive test performance. An optional test of olfaction was also offered to respondents at the end of the interview.

Informant interviews to aid in the diagnosis of cognitive decline and dementia are a mainstay of the typical clinical assessment for dementia and identified as a key source of information for accurate diagnostic classification in recent diagnostic criteria [31]. Building on the ADAMS methodology, the HCAP included an informant interview using several previously validated instruments to provide key information on the respondent's prior and current cognitive and physical function, which helps assess whether the respondent has declined from a prior level of function, and whether the respondent requires help with daily activities, two of the core clinical criteria required for a dementia diagnosis [31].

Ideally, the informant interview was completed inperson immediately after completion of the HCAP respondent interview, with the respondent located in another room. We used a response booklet for this interview to accommodate the potentially sensitive nature of the informant's answers. The informant interview was also adapted for telephone and paper administration. In addition to the informant's relationship with the respondent,
68

Neuroepidemiology 2020;54:64-74 DOI: $10.1159 / 000503004$
Langa/Ryan/McCammon/Jones/Manly/ Levine/Sonnega/Farron/Weir 
Table 2. HRS HCAP tests

\begin{tabular}{lll}
\hline a Respondent test & Cognitive domains ${ }^{1}$ assessed & Citation \\
\hline MMSE & O, MemIE, MemDE, Vis, AS, LF \\
HRS-TICS (3 items: Object naming; Naming President) & MemIE & [37, 38] \\
CERAD Word List Learning and Recall - Immediate & MemIE & [39] \\
Semantic Fluency (Animal Naming Test) & LF & AS \\
Letter Cancellation Test & AS & [40-43] \\
Timed Backward Counting Task (from MIDUS) & O, EF, LF & MemDE \\
Community Screening Instrument for Dementia (CSI-D; 4 items) & MemIE \\
CERAD Word List Recall - Delayed & MemR \\
Story recall - Immediate & Vis \\
CERAD Word List - Recognition & EF, AS \\
CERAD Constructional Praxis - Immediate & MemDE \\
SDMT & MemDE \\
CERAD Constructional Praxis - Delayed & MemR \\
Story recall - delayed & EF \\
Story recall - recognition & EF \\
HRS Number Series & EF, AS \\
Raven's Standard Progressive Matrices & {$[40]$} \\
Trail Making Test (Part A and Part B) & {$[40,50]$} \\
\hline
\end{tabular}

The HCAP tests are listed in the order in which they were administered.

${ }^{1}$ Cognitive Domains. O, orientation; MemIE, immediate episodic memory; MemDE, delayed episodic memory; MemR, recognition Memory; Vis, visuospatial; EF, executive functioning; AS, attention/speed; LF, language/fluency.

HRS, Health and Retirement Study; HCAP, Harmonized Cognitive Assessment Protocol; MMSE, Mini-Mental State Examination; SDMT, Symbol Digit Modalities Test.

\begin{tabular}{|c|c|}
\hline b Informant report instruments & Citation \\
\hline Jorm IQCODE & {$[58]$} \\
\hline Blessed Dementia Rating Scale & {$[59]$} \\
\hline CSI-D Cognitive Activities Questionnaire & {$[60]$} \\
\hline 10/66 Dementia Research Group Informant Questionnaire & {$[7]$} \\
\hline
\end{tabular}

the informant interview ascertained whether the respondent had been previously diagnosed with $\mathrm{AD}$, stroke, Parkinson's disease, or memory problems.

\section{Longitudinal Follow-Up of HCAP Respondents}

We will administer a second wave of respondent and informant HCAP data collection in conjunction with the 2020 wave of the HRS to all surviving members of the original HCAP sample and to a new "age-in" random sample of those aged 65-68 in 2020. This will provide new data to assess change in cognitive function, including the incidence of new cognitive impairment and dementia.
The identification of long-term longitudinal cognitive trajectories will also be possible from ongoing biennial cognitive testing within the core HRS interview. Current funding for the HRS will support the HRS and HCAP 2020 waves of data collection, as well as the HRS 2022 wave. Future waves of data collection for both the HRS and HCAP are planned, pending successful renewal of NIA funding.

HCAP subjects will be tracked between the 2016 and 2020 waves, including after their potential entry into nursing homes, with the HRS procedures that are already established and in place for the full HRS sample. First, all 
Table 3. Characteristics of the HRS-HCAP sample

\begin{tabular}{|c|c|c|c|}
\hline & Assessed $(n=3,496)$ & Not assessed $(n=929)$ & Total $(n=4,425)$ \\
\hline \multicolumn{4}{|l|}{ Age, years } \\
\hline $65-74$ & $1,566(45)$ & $415(45)$ & $1,981(45)$ \\
\hline $75-84$ & $1,416(41)$ & $364(39)$ & $1,780(40)$ \\
\hline $85+$ & $514(15)$ & $150(16)$ & $664(15)$ \\
\hline \multicolumn{4}{|l|}{ Gender } \\
\hline Male & $1,401(40)$ & $358(39)$ & $1,759(40)$ \\
\hline Female & $2,095(60)$ & $571(61)$ & $2,666(60)$ \\
\hline \multicolumn{4}{|l|}{ Race/ethnicity } \\
\hline Hispanic & $383(11)$ & $98(11)$ & $481(11)$ \\
\hline Black, non-hispanic & $551(16)$ & $129(14)$ & $680(15)$ \\
\hline White, non-hispanic & $2,483(71)$ & $681(73)$ & $3,164(72)$ \\
\hline All others & $79(2)$ & $21(2)$ & $100(2)$ \\
\hline \multicolumn{4}{|l|}{ Education } \\
\hline$<12$ years & $714(20)$ & $213(23)$ & $927(21)$ \\
\hline 12 years & $1,166(33)$ & $312(34)$ & $1,478(33)$ \\
\hline $13-15$ years & $765(22)$ & $202(22)$ & $967(22)$ \\
\hline$>16$ years & $851(24)$ & $202(22)$ & $1,053(24)$ \\
\hline \multicolumn{4}{|l|}{ Residence } \\
\hline Community & $3,374(97)$ & $871(94)$ & $4,245(96)$ \\
\hline Nursing home & $122(3)$ & $58(6)$ & $180(4)$ \\
\hline \multicolumn{4}{|c|}{ Respondent type (HRS 2016 interview) } \\
\hline Self & $3,283(94)$ & $800(86)$ & $4,083(92)$ \\
\hline Proxy & $213(6)$ & $129(14)$ & $342(8)$ \\
\hline \multicolumn{4}{|l|}{ US census region } \\
\hline Northeast & $493(14)$ & $176(19)$ & $669(15)$ \\
\hline Midwest & $825(24)$ & $238(26)$ & $1,063(24)$ \\
\hline South & $1,544(44)$ & $362(39)$ & $1,906(43)$ \\
\hline West & $633(18)$ & $153(16)$ & $786(18)$ \\
\hline
\end{tabular}

Values in parentheses are unweighted column percentages.

HRS, Health and Retirement Study; HCAP, Harmonized Cognitive Assessment Protocol.

respondents are asked to provide the name, address, and phone number for 2 individuals who may be contacted in the event that the respondent cannot be reached. Social Security numbers are also collected for tracking purposes. If efforts to reach a contact person fail, a combination of national directories/registries, internet resources, and local sources (e.g., voter registration records, motor vehicle registration, local Department of Health and Human Services office) are employed.

\section{Characteristics of the HCAP Sample}

Characteristics of the HRS respondents randomly selected for the HCAP sample are shown in Table 3, including the 3,496 respondents (79\%) who were assessed (column 1) and 929 (21\%) who were not assessed (column 2). The reasons for not being assessed were refusal to partici- pate $(48 \%)$, unable to contact $(8 \%)$, deceased $(8 \%)$, and other reasons (36\%). The HCAP respondents who were assessed did not differ significantly from nonrespondents in age, sex, race/ethnicity, or educational attainment, but were less likely to have lived in a nursing home $(p<0.001)$, less likely to have been represented by a proxy during the 2016 HRS interview $(p<0.001)$, and more likely to live in the southern United States $(p<0.001)$.

\section{Development of an HCAP Diagnostic Algorithm for $\mathrm{MCl}$ and Dementia}

The HCAP research group is currently developing a diagnostic algorithm that will make use of HCAP respondent and informant data to assign a research diagnosis of normal, MCI, or dementia for each HCAP respondent. The details of this algorithm and the resulting diagnostic 
classifications will be published in a future paper and will be included in future releases of the HCAP data.

As a successor to the ADAMS, the HCAP is not only less expensive, but its diagnostic approach is well-suited to a population-based study due to a reproducible empirical algorithm developed from HCAP data. This will allow diagnoses to be assigned in a reproducible and comparable way with data collected at different points in time or in different countries, thereby increasing international comparability, since case ascertainment will be based on cognitive performance data, functional impairment data, and informant report information collected similarly across the HCAP international studies. While developing comparable and reproducible diagnostic classifications across the HCAP studies, especially for the intermediate state of MCI, presents fundamental measurement challenges, the use of modern psychometric measurement models can accommodate bias in cognitive assessment if such bias can be detected to diminish cultural, and/or language effects in cognitive testing. The use of psychometric measurement models will also allow for case ascertainment ratings to be made on a common metric of measurement, and the identification of impairment thresholds that are equivalent in different samples. This will result in more fair international comparisons. Coupled with the public release of all the raw scores from the cognitive tests and informant reports, researchers will also be able to develop their own diagnostic algorithms, in addition to those developed by the HRS-HCAP team of investigators.

\section{Potential Uses of the HCAP Data}

\section{Assess U.S. Trends in Incidence and Prevalence of \\ MCI and Dementia}

The detailed HCAP data, combined with the cognitive measures collected longitudinally at each HRS core wave, will provide expanded opportunities to track the incidence and prevalence of MCI and dementia in a representative sample of older U.S. adults, as well as individual cognitive trajectories of HRS respondents. The second wave of HCAP data (2020) will increase the sample size and statistical power for identifying incident cases of MCI and dementia. Incident cases provide an important window into the potential factors leading to MCI and dementia through genetic, medical, behavioral, social, and environmental pathways. Risk factors for incident cognitive impairment may differ from factors that predict prevalent cognitive impairment, since prevalence depends both on incidence of disease as well as life-expectancy with the dis- ease. Some risk factors may influence both prevalence and incidence, but the magnitude of their effects may differ. Incidence data are particularly relevant for understanding racial and socioeconomic disparities in MCI and dementia because incidence data address the critique that disparities are the result of bias in measurement or threshold for diagnosis. Additionally, incidence data may increase confidence in causal relationships and the identification of intermediate variables on the causal pathway between race/ethnicity or socioeconomic status and MCI or dementia. Finally, incidence data are useful for identifying the causes of trends in dementia over time, and potential interaction of these causes with race and sex.

\section{International Comparisons}

Harmonized cognitive assessments that support valid international comparisons of dementia incidence and prevalence among the HRS family of longitudinal surveys will also facilitate opportunities to better identify the biological, medical, social, and environmental factors that affect the risk for cognitive decline and dementia across the life course. Similarly, the HRS and its international partners provide an excellent platform for tracking changes in the prevalence of MCI and dementia over time, as a function of country-specific changes in demographics, risk and protective factors (e.g., cardiovascular disease, levels of education), and new medical and/or social policy interventions.

Table 4 shows the overlap of tests that were administered in the 5 international HCAP studies that have been fielded to date. The HCAP research teams from each of these studies will be collaborating on the development of comparable algorithms for diagnosing MCI and dementia across the studies. The HCAP data from all countries will be publicly available so that other researchers will also be able to develop their own methods for data comparison and harmonization.

\section{Linkage of HRS-HCAP Data to Biomarker, Genetic, and Physical Measures}

The HRS has collected an increasing number of biological and physical performance measures that, when combined with HCAP data, will provide unique opportunities for identifying possible predictors of cognitive impairment and dementia. Beginning in 2006, dried blood spot samples were collected from HRS respondents for determination of total cholesterol, HDL cholesterol, glycosylated hemoglobin, C-reactive protein, and Cystatin C [32]. In 2016, venous blood samples were obtained for the first time to allow a much wider range of blood biomarkers to be assayed. A 
Table 4. HCAP test harmonization across studies

\begin{tabular}{|c|c|c|c|c|c|c|c|c|c|c|}
\hline Respondent test & HRS & ELSA & HAALSI & LASI & MHAS & CHARLS & ADAMS & ADNI & ROS/MAP & $10 / 66$ \\
\hline MMSE & $\mathrm{Y}$ & $\mathrm{Y}$ & $\mathrm{H}$ & $\mathrm{H}$ & $\mathrm{H}$ & $\mathrm{Y}$ & $\mathrm{Y}$ & $\mathrm{Y}$ & $\mathrm{Y}$ & $\mathrm{H}$ \\
\hline HRS-TICS ( 3 items: Object naming; Naming President) & $\mathrm{Y}$ & $\mathrm{Y}$ & $\mathrm{Y}$ & $\mathrm{Y}$ & $\mathrm{Y}$ & $\mathrm{Y}$ & $\mathrm{Y}$ & & & \\
\hline CERAD Word List Learning and Recall - Immediate & $\mathrm{Y}$ & $\mathrm{Y}$ & $\mathrm{Y}$ & $\mathrm{Y}$ & $\mathrm{Y}$ & $\mathrm{Y}$ & $\mathrm{Y}$ & $\mathrm{Y}$ & $\mathrm{Y}$ & $\mathrm{Y}$ \\
\hline Semantic Fluency (Animal Naming Test) & $\mathrm{Y}$ & $\mathrm{Y}$ & $\mathrm{Y}$ & $\mathrm{Y}$ & $\mathrm{Y}$ & $\mathrm{Y}$ & $\mathrm{Y}$ & $\mathrm{Y}$ & $\mathrm{Y}$ & $\mathrm{Y}$ \\
\hline Letter Cancellation Test & $\mathrm{Y}$ & $\mathrm{Y}$ & $\mathrm{H}$ & $\mathrm{H}$ & $\mathrm{H}$ & & & & & \\
\hline Timed Backward Counting Task (from MIDUS) & $\mathrm{Y}$ & $\mathrm{Y}$ & $\mathrm{Y}$ & & $\mathrm{H}$ & & & & & \\
\hline Community Screening Instrument for Dementia (CSI-D; 4 items) & $\mathrm{Y}$ & $\mathrm{Y}$ & $\mathrm{Y}$ & $\mathrm{Y}$ & $\mathrm{Y}$ & $\mathrm{Y}$ & & & & $\mathrm{Y}$ \\
\hline CERAD Word List Recall - Delayed & $\mathrm{Y}$ & $\mathrm{Y}$ & $\mathrm{Y}$ & $\mathrm{Y}$ & $\mathrm{Y}$ & $\mathrm{Y}$ & $\mathrm{Y}$ & $\mathrm{Y}$ & $\mathrm{Y}$ & $\mathrm{Y}$ \\
\hline Story recall - Immediate & $\mathrm{Y}$ & $\mathrm{Y}$ & $\mathrm{Y}$ & $\mathrm{Y}$ & $\mathrm{Y}$ & & $\mathrm{H}$ & & $\mathrm{H}$ & $\mathrm{H}$ \\
\hline CERAD Word List - Recognition & $\mathrm{Y}$ & $\mathrm{Y}$ & $\mathrm{Y}$ & $\mathrm{Y}$ & $\mathrm{Y}$ & $\mathrm{Y}$ & $\mathrm{Y}$ & & $\mathrm{H}$ & \\
\hline CERAD Constructional Praxis - Immediate & $\mathrm{Y}$ & $\mathrm{Y}$ & $\mathrm{Y}$ & $\mathrm{Y}$ & $\mathrm{Y}$ & & $\mathrm{Y}$ & $\mathrm{Y}$ & & \\
\hline SDMT & $\mathrm{Y}$ & $\mathrm{Y}$ & $\mathrm{Y}$ & & $\mathrm{H}$ & & $\mathrm{Y}$ & $\mathrm{Y}$ & $\mathrm{Y}$ & \\
\hline CERAD Constructional Praxis - Delayed & $\mathrm{Y}$ & $\mathrm{Y}$ & $\mathrm{Y}$ & $\mathrm{Y}$ & $\mathrm{Y}$ & & $\mathrm{Y}$ & $\mathrm{Y}$ & & \\
\hline Story recall - Delayed & $\mathrm{Y}$ & $\mathrm{Y}$ & $\mathrm{Y}$ & $\mathrm{Y}$ & $\mathrm{Y}$ & & $\mathrm{H}$ & & $\mathrm{H}$ & \\
\hline Story recall - Recognition & $\mathrm{Y}$ & $\mathrm{Y}$ & $\mathrm{Y}$ & $\mathrm{Y}$ & & & $\mathrm{H}$ & & & \\
\hline HRS Number Series & $\mathrm{Y}$ & $\mathrm{Y}$ & $\mathrm{H}$ & & & $\mathrm{H}$ & & & & \\
\hline Raven's Standard Progressive Matrices & $\mathrm{Y}$ & $\mathrm{Y}$ & $\mathrm{Y}$ & $\mathrm{Y}$ & & & & & $\mathrm{Y}$ & \\
\hline Trail Making Test (Part A and B) & $\mathrm{Y}$ & $\mathrm{Y}$ & $\mathrm{H}$ & & & & $\mathrm{Y}$ & $\mathrm{Y}$ & & \\
\hline Coverage of HRS-HCAP Tests (\% Y) & 100 & 100 & 78 & 67 & 56 & 39 & 56 & 44 & 33 & 22 \\
\hline $\begin{array}{l}\text { Coverage of HRS-HCAP Tests (\% linear composite score } \\
\text { variance explained by Y tests) }\end{array}$ & 100 & 100 & 95 & 96 & 93 & 87 & 95 & 94 & 92 & 78 \\
\hline Cognitive domain & & & & & & & & & & \\
\hline Attention/speed & $\bullet$ & $\bullet$ & O & O & O & O & $\mathrm{O}$ & O & O & O \\
\hline Memory & $\bullet$ & $\bullet$ & O & O & 0 & O & O & O & O & O \\
\hline Executive function & $\bullet$ & $\bullet$ & O & O & 0 & O & O & O & O & O \\
\hline Language/fluency & $\bullet$ & $\bullet$ & O & O & O & $\bullet$ & $\bullet$ & O & 0 & O \\
\hline Visouspatial & $\bullet$ & $\bullet$ & O & O & 0 & O & $\bullet$ & $\bullet$ & O & O \\
\hline
\end{tabular}

$\mathrm{Y}$, same test items administered; $\mathrm{H}$, similar test items administered.

-, full coverage of HRS-HCAP cognitive domain (all are Y).

$\mathrm{O}$, partial coverage of HRS-HCAP cognitive domain (not all are $\mathrm{Y}$, but at least 1 test in domain is $\mathrm{Y}$ or $\mathrm{H}$ ).

Studies are: HCAP, Harmonized Cognitive Assessment Protocol; HRS, Health and Retirement Study; ELSA, English Longitudinal Study of Ageing; CHARLS, China Health and Retirement Longitudinal Study; LASI, Longitudinal Aging Study in India; MHAS, Mexican Health and Aging Study; HAALSI, Health and Aging in Africa: Longitudinal Studies in 3 INDEPTH Communities; ADAMS, Aging, Demographics, and Memory Study; ADNI, Alzheimer's Disease Neuroimaging Initiative; ROS/MAP, Rush Religious Orders Study/Memory and Aging Project; 10/66, 10/66 Dementia Research Group Studies; MMSE, Mini-Mental State Examination; SDMT, Symbol Digit Modalities Test.

detailed description of the HRS venous blood sample collection and a full list of the HRS blood biomarker data are available at the HRS website [32]. A venous blood sample is available for about $70 \%$ of the HCAP sample.

The HRS has genotyped nearly 20,000 respondents who provided DNA samples between 2006 and 2012. The HRS genotyping was performed by the NIH Center for Inherited Disease Research, using the Illumina Human Omni-2.5 Quad beadchip, with coverage of approximately 2.5 million single nucleotide polymorphisms. Especially relevant to HCAP, genotype information relevant to $\mathrm{AD}$, including Apolipoprotein E genotype, is included in the HRS genetic data. Polygenic scores that aggregate many individual loci across the human genome and weight them by the strength of their association to produce a single quantitative measure of genetic risk have also been developed for use with HRS data [33]. Polygenic scores for $\mathrm{AD}$ and for general cognition are also currently available for the HRS genetic data. More details on available HRS genetic data and steps to obtain the data can be found at the HRS website [34]. Genotype data are available for about $95 \%$ of the HCAP sample.

Since 2006, the HRS has also collected a number of physical measures that are relevant to brain health and the risk for cognitive decline, including blood pressure, lung function, hand grip strength, balance tests, a timed walk, height, weight, and waist circumference [35]. In 2016, a test of hearing was administered for the first time.

Finally, recognizing the growing scientific interest and focus on neuroimaging as a strategy for identifying individuals with brain pathologies indicative of $\mathrm{AD} / \mathrm{AD}$-related dementias [36], the HRS is teaming with researchers 
from the AD Neuroimaging Initiative (ADNI) to perform a pilot study of neuroimaging in a subsample of 100 HCAP respondents. This project, funded by the U.S. Alzheimer's Association, will perform structural MRI and amyloid PET imaging in the HCAP sub-sample. The goal of the study is to assess the feasibility of neuroimaging in a nationally distributed sample of older adults, with the hope of recruiting a study sample that is more representative of the U.S. population (by race/ethnicity, educational attainment, SES, and health status) compared to prior volunteer samples. Data collection for the HRS neuroimaging pilot study is expected to be completed in 2020 .

\section{Obtaining HRS-HCAP Data}

HRS-HCAP data were released in January 2019 and are publicly available at the HRS website (http://hrsonline.isr.umich.edu/index.php? $\mathrm{p}=$ shoavail\&iyear $=\mathrm{ZU}$ ).

\section{Acknowledgments}

The authors thank Eva Leissou for supervising the HCAP field work and Kelsey Zimmerman for help with manuscript preparation.

\section{Statement of Ethics}

Study subjects and their informants provided written informed consent to participate in the HCAP. The HRS and HCAP study protocols were approved by the University of Michigan Institutional Review Board.

\section{Disclosure Statement}

The authors have no conflicts of interest to declare.

\section{Funding Sources}

The NIA provided funding for the HRS (U01 AG009740) and the HCAP (U01 AG058499), which are performed at the Institute for Social Research, University of Michigan. The NIA did not participate in the preparation of the manuscript, nor the decision to submit for publication.

\section{Author Contributions}

D.R.W., K.M.L., L.H.R., and R.M.: conception or design of the study. D.R.W. and K.M.L.: obtained funding. All authors: acquisition, analysis, or interpretation of data, revising the manuscript for important intellectual content. K.M.L.: drafting the manuscript.

\section{References}

1 Prince MJ, WImo A, Ali G, Wu Y, Prina M. World Alzheimer Report 2015: The Global Impact of Dementia. London: Alzheimer's Disease International; 2015 [cited January 29, 2019]. Available from: https://www.alz.co.uk/ research/world-report-2015.

2 Hurd MD, Martorell P, Delavande A, Mullen KJ, Langa KM. Monetary costs of dementia in the United States. N Engl J Med. 2013 Apr; 368(14):1326-34.

3 Sonnega A, Faul JD, Ofstedal MB, Langa KM, Phillips JW, Weir DR. Cohort Profile: the Health and Retirement Study (HRS). Int J Epidemiol. 2014 Apr;43(2):576-85.

4 International Sister Studies|Health and Retirement Study [Internet]. Health and Retirement Study; 2018 [cited February 18, 2019]. Available from: https://hrs.isr. umich.edu/about/international-sisterstudies.

5 Langa KM, Plassman BL, Wallace RB, Herzog AR, Heeringa SG, Ofstedal MB, et al. The Aging, Demographics, and Memory Study: study design and methods. Neuroepidemiology. 2005;25(4):181-91.

6 McArdle J, Rodgers W, Willis R. Cognition and Aging in the USA (CogUSA) 2007-2009 (ICPSR 36053); 2015.
7 Prince M, Ferri CP, Acosta D, Albanese E, Arizaga R, Dewey M, et al. The protocols for the $10 / 66$ dementia research group population-based research programme. BMC Public Health. 2007 Jul;7(1):165.

8 Hendrie HC, Ogunniyi A, Hall KS, Baiyewu $\mathrm{O}$, Unverzagt FW, Gureje $\mathrm{O}$, et al. Incidence of dementia and Alzheimer disease in 2 communities: yoruba residing in Ibadan, Nigeria, and African Americans residing in Indianapolis, Indiana. JAMA. 2001 Feb;285(6):739-47.

9 Brayne C, McCracken C, Matthews FE; Medical Research Council Coginitive Function and Ageing Study (CFAS). Cohort profile: the Medical Research Council Cognitive Function and Ageing Study (CFAS). Int J Epidemiol. 2006 Oct;35(5):1140-5.

10 Wong R, Michaels-Obregon A, Palloni A. Cohort Profile: The Mexican Health and Aging Study (MHAS). Int J Epidemiol. 2017 Apr; 46(2):e2-2.

11 Arokiasamy P, Bloom D, Lee J, Feeney K, Ozolins M. Longitudinal Aging Study in India: Vision, Design, Implementation, and Preliminary Findings. National Academies Press (US); 2012 [cited January 30, 2019]. Available from: https://www.ncbi.nlm.nih. gov/books/NBK109220/.
12 Steptoe A, Breeze E, Banks J, Nazroo J. Cohort profile: the English longitudinal study of ageing. Int J Epidemiol. 2013 Dec;42(6):1640-8.

13 Zhao Y, Hu Y, Smith JP, Strauss J, Yang G. Cohort profile: the China Health and Retirement Longitudinal Study (CHARLS). Int J Epidemiol. 2014 Feb;43(1):61-8.

14 Gómez-Olivé FX, Montana L, Wagner RG, Kabudula CW, Rohr JK, Kahn K, et al. Cohort Profile: Health and Ageing in Africa: a Longitudinal Study of an INDEPTH Community in South Africa (HAALSI). Int J Epidemiol. 2018 Jan;47(3):689-690j.

15 Börsch-Supan A, Brandt M, Hunkler C Kneip T, Korbmacher J, Malter F, et al.; SHARE Central Coordination Team. Data Resource Profile: the Survey of Health, Ageing and Retirement in Europe (SHARE). Int J Epidemiol. 2013 Aug;42(4):992-1001.

16 Park JH, Lim S, Lim JY, Kim KI, Han MK, Yoon IY, et al. An Overview of the Korean Longitudinal Study on Health and Aging. Psychiatry Investig 2007;4:84-95.

17 Korean Longitudinal Study of Aging Website [Internet]. Korean Longitudinal Study of Aging Website; 2016 [cited May 1, 2019]. Available from: https://survey.keis.or.kr/eng/ klosa/klosa01.jsp. 
18 Plassman BL, Langa KM, Fisher GG, Heeringa SG, Weir DR, Ofstedal MB, et al. Prevalence of dementia in the United States: the aging, demographics, and memory study. Neuroepidemiology. 2007;29(1-2):125-32.

19 Plassman BL, Langa KM, Fisher GG, Heeringa SG, Weir DR, Ofstedal MB, et al. Prevalence of cognitive impairment without dementia in the United States. Ann Intern Med. 2008 Mar; 148(6):427-34.

20 Plassman BL, Langa KM, McCammon RJ, Fisher GG, Potter GG, Burke JR, et al. Incidence of dementia and cognitive impairment, not dementia in the United States. Ann Neurol. 2011 Sep;70(3):418-26.

21 Crimmins EM, Kim JK, Langa KM, Weir DR. Assessment of cognition using surveys and neuropsychological assessment: the Health and Retirement Study and the Aging, Demographics, and Memory Study. J Gerontol B Psychol Sci Soc Sci. 2011 Jul;66(suppl 1):i162-71.

$22 \mathrm{Wu}$ YT, Lee HY, Norton S, Chen C, Chen H, $\mathrm{He} \mathrm{C}$, et al. Prevalence studies of dementia in mainland china, Hong Kong and taiwan: a systematic review and meta-analysis. PLoS One. 2013 Jun;8(6):e66252.

23 Gianattasio KZ, Wu Q, Glymour MM, Power MC. Comparison of Methods for Algorithmic Classification of Dementia Status in the Health and Retirement Study. Epidemiology. 2019 Mar;30(2):291-302.

24 Iwashyna TJ, Ely EW, Smith DM, Langa KM. Long-term cognitive impairment and functional disability among survivors of severe sepsis. JAMA. 2010 Oct;304(16):1787-94.

25 Llewellyn DJ, Lang IA, Matthews FE, Plassman BL, Rogers MA, Morgenstern LB, et al. Vascular health, diabetes, APOE and dementia: the Aging, Demographics, and Memory Study. Alzheimers Res Ther. 2010 Jun;2(3):19.

26 Nicholas LH, Bynum JP, Iwashyna TJ, Weir $\mathrm{DR}$, Langa KM. Advance directives and nursing home stays associated with less aggressive end-of-life care for patients with severe dementia. Health Aff (Millwood). 2014 Apr; 33(4):667-74.

27 Nicholas LH, Langa KM, Iwashyna TJ, Weir DR. Regional variation in the association between advance directives and end-of-life Medicare expenditures. JAMA. 2011 Oct; 306(13):1447-53.

28 Bennett DA, Buchman AS, Boyle PA, Barnes LL, Wilson RS, Schneider JA. Religious Orders Study and Rush Memory and Aging Project. J Alzheimers Dis. 2018;64 s1:S161-89.

29 Weir DR, McCammon R, Ryan LH, Langa KM. Cognitive Test Selection for the Harmonized Cognitive Assessment Protocol (HCAP). Ann Arbor (Michigan): Institute for Social Research, University of Michigan; 2014

30 Staff HR. Data Product Detail|Health and Retirement Study [Internet]. Health and Retirement Study; 2019 [cited February 18, 2019]. Available from: http://hrsonline.isr.umich. edu/index.php? $\mathrm{p}=$ shoavail\&iyear $=\mathrm{ZU}$

31 McKhann GM, Knopman DS, Chertkow H, Hyman BT, Jack CR Jr, Kawas CH, et al. The diagnosis of dementia due to Alzheimer's disease: recommendations from the National Institute on Aging-Alzheimer's Association workgroups on diagnostic guidelines for $\mathrm{Al}$ zheimer's disease. Alzheimers Dement. 2011 May;7(3):263-9.

32 Crimmins E, Faul J, Kim J, Guyer H, Langa K, Ofstedal MB, et al. Documentation of Biomarkers in the 2006 and 2008 Health and Retirement Study; 2013.

33 Ware EB, Schmitz LL, Faul JD. HRS Polygenic Scores: 2006-2010 Genetic Data. Ann Arbor, Michigan: Survey Research Center, Institute for Social Research, University of Michigan; 2017 [cited August 15, 2018]. Available from: https://hrs.isr.umich.edu/sites/default/ files/biblio/PGENSCORES_0.pdf.

34 Health and Retirement Study. Genetic Data [Internet]; 2018 [cited August 15, 2018]. Available from: https://hrs.isr.umich.edu/data-products/genetic-data.

35 Crimmins E, Guyer H, Langa K, Ofstedal M, Wallace R, Weir D. Documentation of Physical Measures, Anthropometrics and Blood Pressure in the Health and Retirement Study; 2008.

36 Jack CR Jr, Bennett DA, Blennow K, Carrillo MC, Dunn B, Haeberlein SB, et al.; Contributors. NIA-AA Research Framework: toward a biological definition of Alzheimer's disease. Alzheimers Dement. 2018 Apr;14(4):535-62.

37 Folstein MF, Folstein SE, McHugh PR. "Minimental state". A practical method for grading the cognitive state of patients for the clinician. J Psychiatr Res. 1975 Nov;12(3):189-98.

38 Crum RM, Anthony JC, Bassett SS, Folstein MF. Population-based norms for the MiniMental State Examination by age and educational level. JAMA. 1993 May;269(18):238691.

39 Brandt J, Spencer M, Folstein MF. The telephone interview for cognitive status. Cogn Behav Neurol. 1988;1(2):111-7.

40 Morris JC, Heyman A, Mohs RC, Hughes JP, van Belle G, Fillenbaum G, et al. The consortium to establish a registry for Alzheimer's Disease (CERAD). Part I. Clinical and neuropsychological assessment of Alzheimer's Disease. Neurology. 1989 Sep;39(9):1159-65.

41 Harold G, Barbara B, Kaplan E. The assessment of aphasia and related disorders. Philadelphia: Lippincott Williams \& Wilkins; 2001. Available from https://www.ncbi.nlm. nih.gov/pmc/articles/PMC494503/pdf/jnnpsyc00203-0198c.pdf.

42 Kertesz A. Western Aphasia Battery. San Antonio (TX): The PsychologicalCorporation; 1982.

43 Thurstone LL. Primary mental abilities; 1938.

44 Pam MS. Letter Cancellation Test [Internet]. Psychology Dictionary; 2013 April [cited February 19, 2019]. Available from: https://psychologydictionary.org/letter-cancellationtest/.

45 Agrigoroaei S, Lachman ME. Cognitive functioning in midlife and old age: combined effects of psychosocial and behavioral factors. J Gerontol B Psychol Sci Soc Sci. 2011 Jul; 66(suppl 1):i130-40.
46 Prince $M$, Acosta D, Ferri CP, Guerra M, Huang Y, Jacob KS, et al.; 10/66 Dementia Group. A brief dementia screener suitable for use by non-specialists in resource poor settings-the cross-cultural derivation and validation of the brief Community Screening Instrument for Dementia. Int J Geriatr Psychiatry. 2011 Sep;26(9):899-907.

47 Ofstedal M, Fisher G, Herzog AR, Herzog AR. Documentation of Cognitive Functioning Measures in the Health and Retirement Study; 2005.

48 McArdle JJ, Fisher GG, Kadlec KM. Latent variable analyses of age trends of cognition in the Health and Retirement Study, 1992-2004. Psychol Aging. 2007 Sep;22(3):525-45.

49 Wechsler D. Wechsler Memory Scale - Revised Manual. San Antonio (TX): The Psychological Corporation; 1987.

50 Rosen WG, Mohs RC, Davis KL. A new rating scale for Alzheimer's disease. Am J Psychiatry. 1984 Nov;141(11):1356-64.

51 Smith A. Symbol Digit Modalities Test. Los Angeles (CA): Western Pscychological Services; 1982 [cited February 19, 2019]. Available from https://www.communicate-ed.org. uk/assets/downloads/SDMT_Formula_ Chart_Communicate-ed_2.pdf.

52 Fisher G, McArdle J, McCammon R, Sonnega A, Weir D. New measures of fluid intelligence in the HRS; 2013.

53 Raven J. The Raven's progressive matrices: change and stability over culture and time. Cognit Psychol. 2000 Aug;41(1):1-48.

54 Raven J. The Raven Progressive Matrices: A review of national norming studies and ethnic and socioeconomic variation within the United States. J Educ Meas. 1989;26(1):1-16.

55 Raven J. Manual for Raven's Progressive Matrices and Vocabulary Scales. Research supplement no. 1: The 1979 British standardization of the Standard Progressive Matrices and Mill Hill Vocabulary Scales, together with comparative data from earlier studies in the UK, US, Canada, Germany, and Ireland. Oxford, England: Oxford Psychologists Press/ The Psychological Corporation; 1981.

56 Reitan RM. Trail Making Test: Manual for Administration and Scoring. Tuscon (AZ): Reitan Neuropsychological Laboratory; 1994.

57 Ricker JH, Axelrod BN. Analysis of an Oral Paradigm for the Trail Making Test. Assessment. 1994 Mar;1(1):47-52.

58 Jorm AF. A short form of the Informant Questionnaire on Cognitive Decline in the Elderly (IQCODE): development and cross-validation. Psychol Med. 1994 Feb;24(1):145-53.

59 Blessed G, Tomlinson BE, Roth M. The association between quantitative measures of dementia and of senile change in the cerebral grey matter of elderly subjects. Br J Psychiatry. 1968 Jul;114(512):797-811.

60 Hall KS, Hendrie HH, Brittain HM, Norton JA, Rodgers DD, Prince CS, et al. The development of a dementia screeing interview in two distinct languages. Int J Methods Psychiatr Res. 1993;3:1-28. 